\section{Relative magnitude of apparent misalignment in acute-angle and oblique-line figures}

\section{R. H. DAY and R. G. DICKINSON Monash University \\ Clayton, Victoria 3168, Australia}

In the second of a series of experiments reported earlier (Day, 1973), it was found that the apparent misalignment of two oblique, collinear lines forming the arms of two $45^{\circ}$ acute angles was no greater than that of the two oblique lines alone. It was therefore concluded that the acute angles do not themselves contribute to the misalignment illusion. A more recent experiment by Wenderoth, Beh, and White (1978) did not support this conclusion. It was found that apparent misalignment between a dot and an oblique line forming the arm of a $45^{\circ}$ acute angle was greater than that between the dot and the oblique line alone.

While these outcomes appear inconsistent, the two experiments were different in a number of ways. In Day's experiment, the display was horizontal, the method of adjustment was used, and the angles and lines were derived from the parallel-line figure shown in Figure 1A. In the experiment described by Wenderoth et al., the display was vertical (the face of an oscilloscope), the random interleaved staircase method was used, and the angle, line, and dots were derived from the basic figure shown in Figure 1B. However, since, except for the relative magnitude of misalignment for the acute-angle and single-line figures, the other results reported by Wenderoth et al. were in good agreement with Day's, it seems unlikely that the different displays or procedures were implicated. It is more likely that the differences between the members of the two pairs of figures were involved. The figures used by Day (1973) are shown in Figures $2 \mathrm{~A}$ and $2 \mathrm{~B}$ and those used by Wenderoth et al. (1978) in Figures 2C and 2D. It can be seen that, in Day's experiment, one figure consisted of two acute angles, each with a vertical arm slightly shorter than the oblique and the other of two oblique lines. In the experiment by Wenderoth et al., one figure consisted of a single acute angle and a dot with the vertical arm of the former about three times longer than the oblique. The other figure consisted simply of an oblique line and a dot.

The purpose of the experiment reported here was to establish whether the inconsistency between the two earlier experiments was attributable to differences between the two pairs of figures. Therefore, the two pairs, Figures $2 \mathrm{~A}$ and $2 \mathrm{~B}$ and Figures $2 \mathrm{C}$ and $2 \mathrm{D}$, were essentially the same as those used originally.
Furthermore, in order to identify which feature or combination of features caused the inconsistency (in the event that the earlier results were confirmed), two additional figures were included. These are shown in Figures 2E and 2F. That shown in Figure 2E was intended to reveal, through appropriate comparisons, any effect due to the length of the vertical line and that in Figure 2F any effect due to a dot rather than a line.

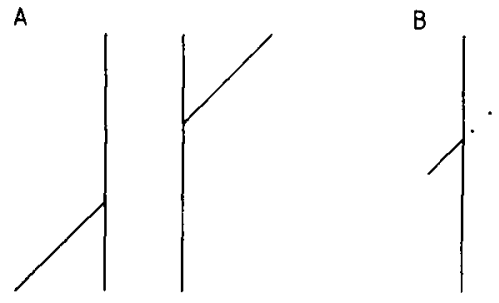

Figure 1. The figures from which the acute-angle and obliqueline figures were derived in the experiments by Day (A) and Wenderoth, Beh, and White (B).

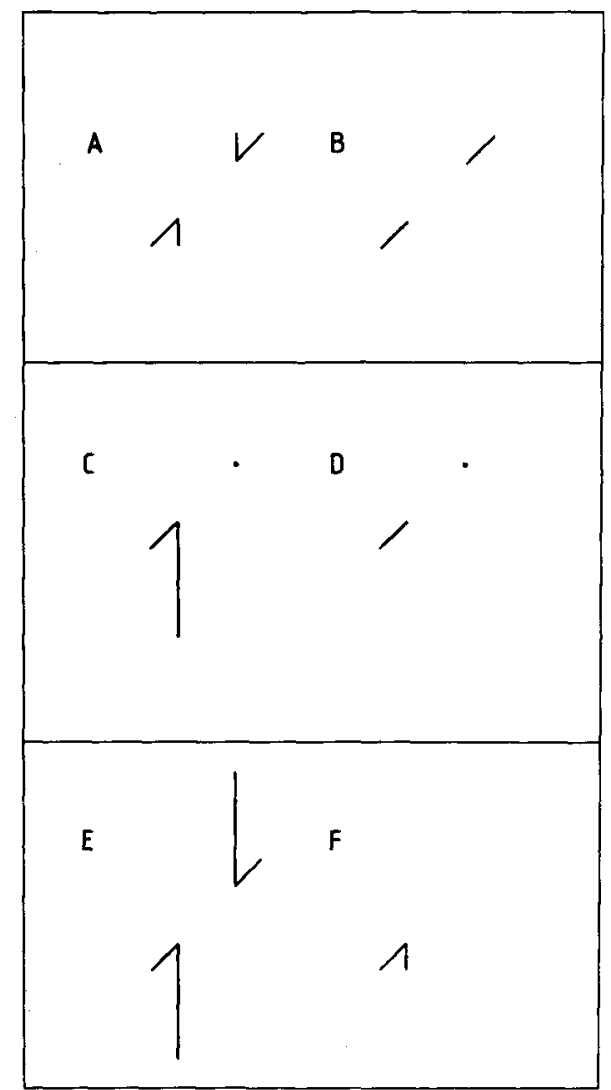

Figure 2. The acuteangle and oblique-line figures. Figures $A$ and $B$ are ensentially the same as those used originally by Day and $C$ and $D$ as those by Wenderoth, Beh, and White. 


\section{METHOD}

\section{Subjects}

There were 12 volunteer, paid subjects, 7 men and 5 women, all of whom were undergraduates, graduate students, or research assistants. None were familiar with the experiment or the stimulus figure.

\section{Apparatus}

The apparatus has been described in detail before (Day, 1973; Day \& Dickinson, 1976). Basically, it consisted of a back. illuminated, circular display area of translucent Plexiglas, carrying the fixed component of the figure on its upper surface, and a transparent rectangular Plexiglas slide, carrying the adjustable component on its lower surface. The circular area at the center of which the figures appeared was $20 \mathrm{~cm}$ in diameter. The subjects looked down on the display with their chins on a bar so that the viewing distance was $57 \mathrm{~cm}$. At this distance, $1 \mathrm{~cm}$ at the display subtended a visual angle of $P$. The adjustable slide could be moved toward and away from the subject by the subject himself in order to bring the two oblique lines or the oblique line and the dot into apparent alignment.

\section{Stimulus Figures}

The stimulus figures are represented in Figure 2. Those in Figures 2A and 2B were derived from Figure $1 \mathrm{~A}$ and were essentially the same as those used by Day (1973). The vertical lines were $14 \mathrm{~mm}$ long, and the oblique lines $20 \mathrm{~mm}$. The gap between the two obliques was $42 \mathrm{~mm}$. The figures shown in Figures $2 \mathrm{C}$ and 2D were derived from Figure $1 \mathrm{~B}$ and closely resembled those used by Wenderoth et al. (1978). The oblique lines were $20 \mathrm{~mm}$ long, and the vertical line, $60 \mathrm{~mm}$. The dots were slightly less than $1 \mathrm{~mm}$ in diameter. The gap between line and dot was $42 \mathrm{~mm}$. The figures shown in Figures $2 \mathrm{E}$ and $2 \mathrm{~F}$ were included for the sake of completeness. The former simply consisted of two acute angles with long verticals, as in Figure $2 \mathrm{C}$ and the latter of one acute angle the same as that in Figures $2 \mathrm{~A}$ and a dot.

In each figure the component on the left was fixed. That on the right could be moved towards or away from the subject in order to align the oblique line or the dot with the oblique line of the fixed component.

\section{Procedure}

The subject's task throughout was to adjust the moving element of each figure so that it appeared perfectly aligned with the oblique line of the fixed element. The six figures were presented in a random order. Four adjustments were made for each figure, two ascending $(A)$ and two descending (D). Half the adjustments were made in the order ADDA and half in the order DAAD. The four adjustments for one figure were completed before the presentation of the next. The score was the mean misalignment in millimeters between the two obliques or between the oblique and the dot. Misalignments were measured along a line parallel to the main axis of the figures through the inner end of the variable oblique or through the dot.

\section{RESULTS}

The mean misalignments for the six figures are shown together with their standard deviations in Table 1. Inspection of these means shows straightaway that there is little difference between those for the figures used originally by Day (Figures $2 \mathrm{~A}$ and 2B) but a marked difference between those for the figures used by Wenderoth et al. (Figures 2C and 2D). For the latter pair, the mean misalignment for the acute-angle figure is over three times greater than that consisting of an oblique line and a dot. The
Table 1

Means (in Millimeters) and Standard Deviations of Misalignments for the Six Figures Shown in Figure 2

\begin{tabular}{lcccccc}
\hline & A & B & C & D & E & F \\
\hline $\bar{X}$ & 1.33 & 1.60 & 5.12 & 1.97 & 4.34 & 3.47 \\
SD & 2.50 & 1.51 & 2.51 & 1.01 & 1.63 & 2.08 \\
\hline
\end{tabular}

Note-Misalignments were measured along the vertical axis of the figures.

means for the figures represented in Figures $2 \mathrm{E}$ and $2 \mathrm{~F}$ suggest also that both the longer vertical arm in the acute angle and a single dot rather than an oblique line give rise to a greater misalignment.

The data have been statistically analyzed in two ways. An analysis of variance to test for differences between the means, and planned comparisons to test for the differences of greatest interest.

The analysis of variance indicated that there was a significant difference between the means $[F(5,55)=$ 10.57, $\mathrm{p}<.01$ ]. Planned comparisons showed that, while the difference in mean misalignment between the figures shown in Figures $2 \mathrm{~A}$ and $2 \mathrm{~B}$ was not significant $[F(1,55)<1]$, that between the figures shown in Figures $2 C$ and $2 D$ was $[F(1,55)=21.23$, $\mathrm{p}<.01$ ], thus confirming the results for the figures used by Day (1973) and by Wenderoth et al. (1978). Two further planned comparisons showed that the misalignments between the figures shown in Figures $2 \mathrm{~A}$ and $2 \mathrm{E}$ and between those shown in Figures $2 \mathrm{~A}$ and $2 F$ were also significant $[F(1,55)=19.48$, $\mathrm{p}<.01 ; \mathrm{F}(1,55)=9.88, \mathrm{p}<.01$, respectively]. These outcomes implicate both the length of the vertical line of the acute-angle figure and the form of the adjustable element, oblique line or dot, in the difference between the findings in the earlier experiments.

\section{DISCUSSION}

The main outcome of this experiment is the resolution of an apparent inconsistency between the results reported by Day (1973) and Wenderoth et al. (1978). The results confirm both sets of earlier findings and indicate that the differences are attributable to the particular figures used in the original experiments. A secondary outcome is that both the length of the vertical arm in the acute-angle figures and the form of the adjustable component, an oblique line or a dot, contribute to the size of the apparent misalignment between the obliquely disposed elements. The effect is greater with the longer arm and with an adjustable dot.

One further, somewhat speculative, point is worth making in conclusion. Inspection of the figures shown in Figure 2 clearly shows how such properties as the length of the vertical lines relative to the presence of an oblique or a dot determines the overall configuration. For example, the two acute angles with 
long verticals result in a figure with a long axis oriented vertically. The figure consisting of two acute angles with shorter verticals has more the appearance of two arrowheads pointing toward each other. It is conceivable that such total configurations determine the magnitude and possibly the direction of apparent misalignment. For example, in Figures $2 \mathrm{C}$ and $2 \mathrm{E}$, with a distinct vertical axis it is possible that the misalignment of the two obliquely disposed elements relative to the axis affects their apparent collinearity. That is, perceived alignment may be relative, as are perceived size, orientation, and velocity.

\section{REFERENCES}

DAY, R. H. The Poggendorff illusion with obtuse and acute angles. Perception \& Psychophysics, 1973, 14, 590-596.

DAY, R. H., \& Drckinson, R. G. The components of the Poggendorff illusion. British Journal of Psychology, 1976, 67, 537-552.

Wenderoth, P., Beh, H., \& White, D. Alignment errors to both ends of acute- and obtuse-angle arms. Perception \& Psychophysics, 1978, 6, 475-482.

(Received and accepted for publication December 22, 1978.)

\section{ANNOUNCEMENT \\ Boston University Conference on Language Development}

The Boston University School of Education announces the Fourth Annual Boston University Conference on Language Development, to be held September 14-15, 1979, at the George Sherman Union, 775 Commonwealth Avenue. Papers in the areas of first and second language acquisition, disorders of language, sign language, and the development of reading and written language will be considered for presentation at the conference. The deadline for submission of abstracts is June 1, 1979. To obtain abstract guidelines and information on preregistration and local arrangements, contact: Language Development Conference, Box F, Boston University, Boston, Massachusetts 02215. Phone: (617) 353-3085. 